\title{
HSV management in pregnancy at a joint antenatal-genitourinary clinic in a large maternity hospital in Dublin, Ireland - a model of care. \\ N Lynn ${ }^{1}$, S Murphy ${ }^{1}$, O Cunningham², A Lynam¹, F Lyons ${ }^{1}$, F Mulcahy ${ }^{1}$ \\ ${ }^{1}$ GUIDe Clinic. St. James's Hospital, Dublin ${ }^{2}$ The Coombe Women and Infants University Hospital, Dublin.
}

\section{Introduction:}

The ultimate goal of HSV management in pregnancy is to prevent perinatal transmission and, where possible, to facilitate a vaginal delivery'. The GUIDE clinic in St. James's Hospital provides a satellite genito-urinary medicine service to The Coombe Women and Infants University Hospital, Dublin. Patient's were referred to the clinic if they reported prior history of HSV or had an outbreak during that pregnancy

\section{Results:}

Between May 2013 and Feb 2017, 107 women were referred to this service for management of HSV in 108 pregnancies. Median gestation at referral (82/108) 23/40 (range 2-39/40). The average age was 33 (range 18-45).

Data on country of origin available on 100\%- figure 1 .

$\square$ Europe
$\square$ Asia
$\square$ Africa
$\square$ Russia
$\square$ South Africa
$\square$ America
$\square$ South America

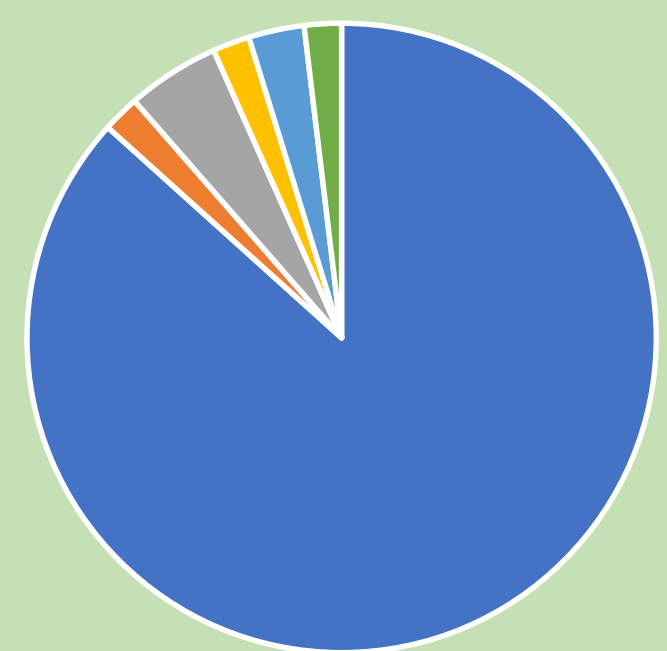

Figure 1; Country of Origin

$9(8 \%)$ women were HIV+.

NAATs testing for Chlamydia trachomatis and Neisseria gonorrhoea done in 63\% (69) pregnancies, $100 \%$ negative.

$100 \%(n=107)$ were asked about history of HSV at booking;

- $76 \%$ (82) reported a history of HSV prior to booking

- $23 \%(25)$ no history of HSV a booking.

89\% (96) had HSV type-specific serology sent -figure 2.

Of those who were HSV type $1+$ in serum (75), $25 \%$ (19) had genital swabs for HSV PCR sent and 11 were positive.

$90 \%$ (96) reported genital lesions.

$5 \%(6)$ reported lesions in extragenital sites.

\section{Outbreaks:}

Last outbreak recorded in 86\% (93) pregnancies.

Outbreak frequency recorded in $88 \%$ (63) pregnancies;

- $30 \%$ (32) pregnancies had outbreak in that pregnancy.

- 6\% (6)had primary infection during current pregnancy, none in third trimester.
(C Combe Women a Infants University Hospital

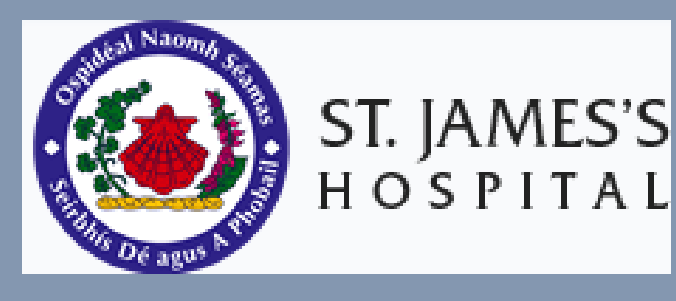

\section{Methods:}

Data was collected from antenatal charts of 108 pregnancies. Descriptive column statistics were used in excel for data analysis.

\section{Figure 2: HSV IgG positive in serum}

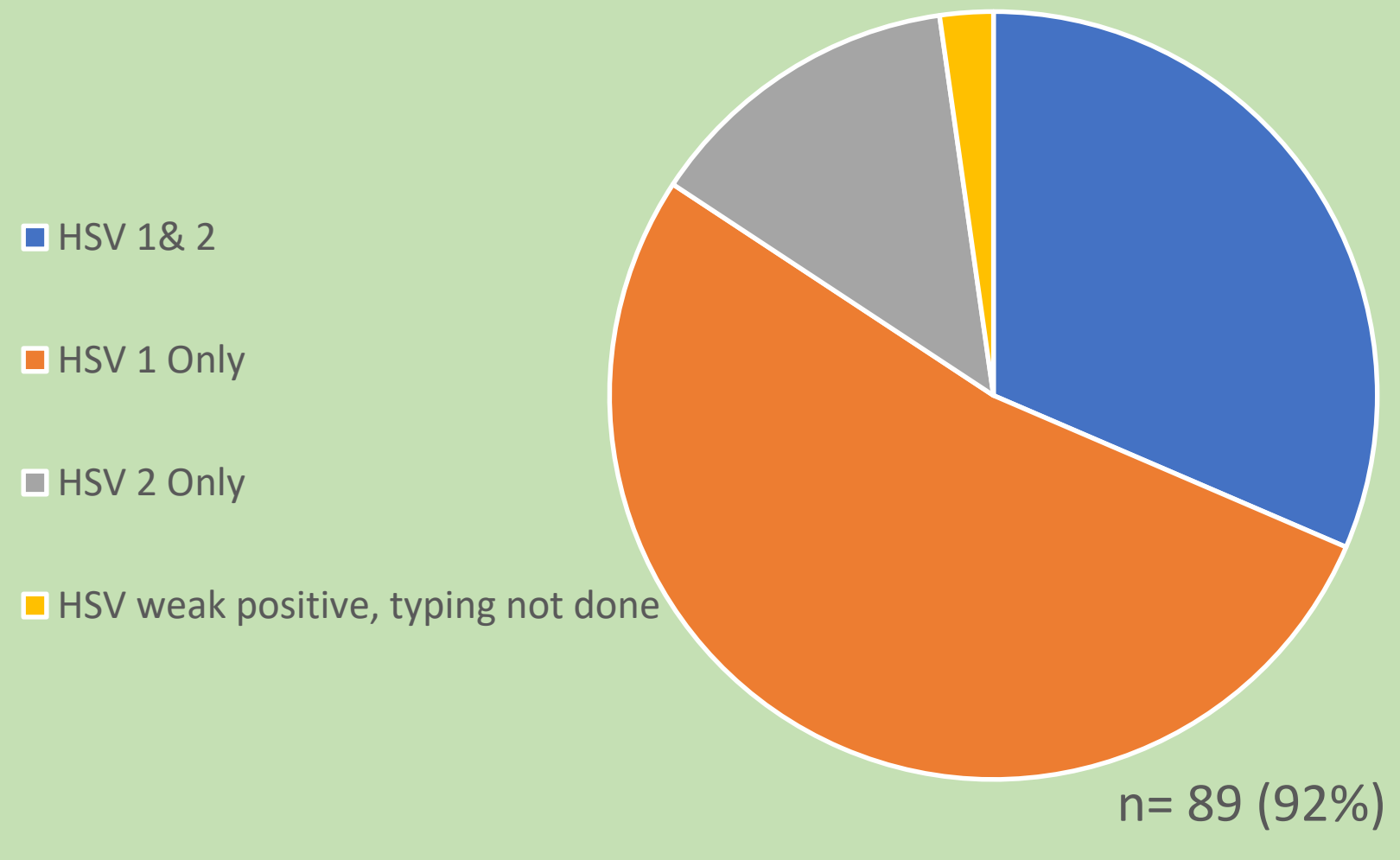

\section{Management:}

Mean gestation starting prophylaxis was 36/40 (range20-39).

$63 \%$ (68) women received antiviral medication;

- 67 were treated with valaciclovir in 66 pregnancies

- 1 woman received acyclovir.

- $24 \%(26)$ patients were not started on prophylaxis.

Median gestation at delivery of 84 pregnancies 39/40 (range 29-41).

\section{Mode of Delivery:}

Data available for $76 \%$ (82) pregnancies:

- $71 \%$ (59) vaginal deliveries

- $29 \%$ (24) lower segment caesarean sections, none for HSV.

To date no cases of perinatal HSV transmission have been reported.

\section{Conclusion:}

There is good compliance with Irish guidelines on HSV management in pregnancy ${ }^{2}$. HSV2 remains an issue. This combined clinic facilitates good compliance with standard guidelines for HSV management in pregnancy. This model of care should be available across all antenatal settings 